\title{
An event study on broadband electric field noises and electron distributions in the lunar wake boundary
}

\author{
Masaki N. Nishino ${ }^{1 *}\left(\mathbb{0}\right.$, Yoshiya Kasahara², Yuki Harada ${ }^{3}$, Yoshifumi Saito ${ }^{1}$, Hideo Tsunakawa', \\ Atsushi Kumamoto ${ }^{4}$, Shoichiro Yokota ${ }^{5}$, Futoshi Takahashi ${ }^{6}$, Masaki Matsushima $^{7}$, Hidetoshi Shibuya ${ }^{8}$, \\ Hisayoshi Shimizu ${ }^{9}$, Yukinaga Miyashita ${ }^{10,11}$, Yoshitaka Goto ${ }^{2 \wedge}$ and Takayuki Ono ${ }^{4 \wedge}$
}

\begin{abstract}
Wave-particle interactions are fundamental processes in space plasma, and some plasma waves, including electrostatic solitary waves (ESWs), are recognised as broadband noises (BBNs) in the electric field spectral data. Spacecraft observations in recent decades have detected BBNs around the Moon, but the generation mechanism of the BBNs is not fully understood. Here, we study a wake boundary traversal with BBNs observed by Kaguya, which includes an ESW event previously reported by Hashimoto et al. Geophys Res Lett 37:L19204 https://doi.org/10.1029/2010GL0445 29 (2010). Focusing on the relation between BBNs and electron pitch-angle distribution functions, we show that upward electron beams from the nightside lunar surface are effective for the generation of BBNs, in contrast to the original interpretation by Hashimoto et al. Geophys Res Lett 37:L19204 https://doi.org/10.1029/2010GL044529 (2010) that high-energy electrons accelerated by strong ambipolar electric fields excite ESWs in the region far from the Moon. When the BBNs were observed by the Kaguya spacecraft in the wake boundary, the spacecraft's location was magnetically connected to the nightside lunar surface, and bi-streaming electron distributions of downward-going solar wind strahl component and upward-going field-aligned beams (at $\sim 124 \mathrm{eV}$ ) were detected. The interplanetary magnetic field was dominated by a positive $B_{Z}$ (i.e. the northward component), and strahl electrons travelled in the antiparallel direction to the interplanetary magnetic field (i.e. southward), which enabled the strahl electrons to precipitate onto the nightside lunar surface directly. The incident solar wind electrons cause negative charging of the nightside lunar surface, which generates downward electric fields that accelerate electrons from the nightside surface toward higher altitudes along the magnetic field. The bidirectional electron distribution is not a sufficient condition for the BBN generation, and the distribution of upward electron beams seems to be correlated with the BBNs. Ambipolar electric fields in the wake boundary should also contribute to the electron acceleration toward higher altitudes and further intrusion of the solar wind ions into the deeper wake. We suggest that solar wind ion intrusion into the wake boundary is also an important factor that controls the BBN generation by facilitating the influx of solar wind electrons there.
\end{abstract}

\footnotetext{
*Correspondence: nishino@stp.isas.jaxa.jp

^Yoshitaka Goto and Takayuki Ono deceased on 8 April 2020 and 21 December 2013

${ }^{1}$ Institute of Space and Astronautical Science, Japan Aerospace Exploration Agency, 3-1-1 Yoshinodai, Chuo-ku, Sagamihara, Kanagawa 252-5210, Japan

Full list of author information is available at the end of the article
} original author(s) and the source, provide a link to the Creative Commons licence, and indicate if changes were made. The images or other third party material in this article are included in the article's Creative Commons licence, unless indicated otherwise in a credit line to the material. If material is not included in the article's Creative Commons licence and your intended use is not permitted by statutory regulation or exceeds the permitted use, you will need to obtain permission directly from the copyright holder. To view a copy of this licence, visit http://creativecommons.org/licenses/by/4.0/. 
Keywords: Lunar plasma environment, Wave-particle interaction, Solar wind, Lunar wake, Broadband electric field noise

\section{Graphical Abstract}

Broadband electric field noises and electron pitch-angle distribution in the lunar wake boundary

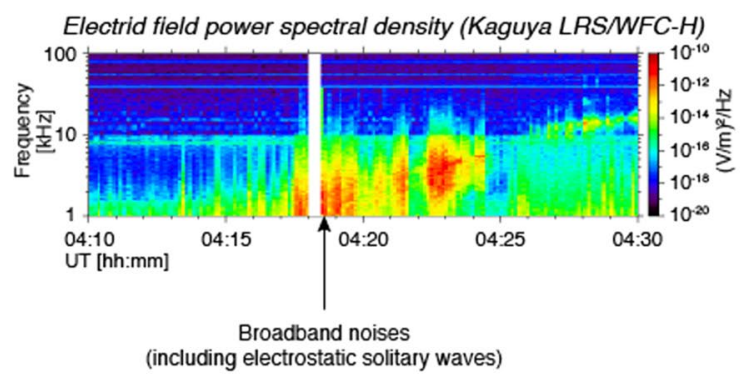

Upward-going electron beam
Electron distribution (Kaguya PACE+LMAG)

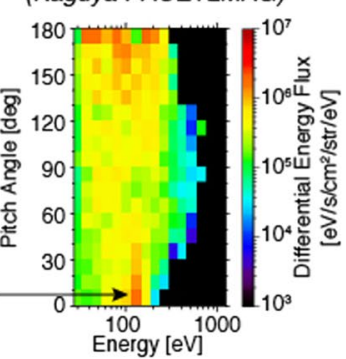

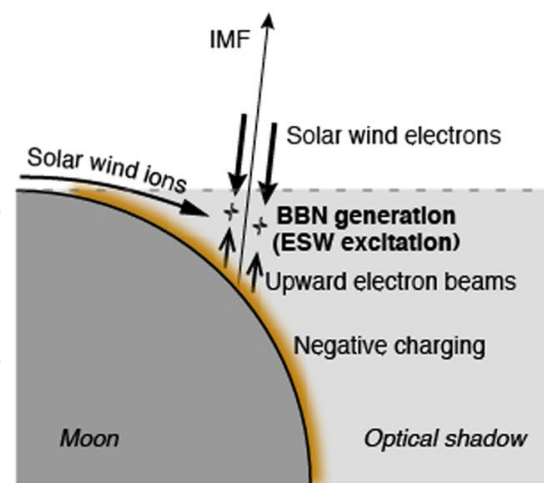

\section{Introduction}

Direct observations in orbit around the Moon in the last decades have revealed the plasma environment including plasma waves that are excited due to interactions between the Moon and surrounding space plasmas (e.g. Harada and Halekas 2016; Nakagawa 2016). These waves are categorised into electrostatic waves and electromagnetic waves, both of which are detected around the Moon. One of the electrostatic waves present around the Moon is the electrostatic solitary wave (ESW) derived from wave-particle interactions. The ESWs, also called electrostatic solitary structures, are nonlinear, bipolar electric field pulses that appear in the electric field waveform data (e.g. Pickett 2021). Historically, ESWs were observed in the plasma sheet boundary layer of the Earth's magnetotail (Matsumoto et al. 1994), and their substances are known to be electron phase-space holes (Omura et al. 1994). On the other hand, ESWs observed in the turbulent magnetosheath downstream of the bow shock is likely to be ion phase-space holes (Holmes et al. 2017). These ESWs are recognised as BBNs in the frequency-time spectrograms of electric field data, which is mainly attributed to the higher harmonics caused by the nature of isolated bipolar pulses (Matsumoto et al. 1994).

Since most solar wind plasmas are absorbed by the dayside lunar surface, a tenuous region called 'lunar wake' is formed on the lunar nightside (e.g. Schubert and Lichtenstein 1974; Ogilvie et al. 1996). Solar wind plasma refilling into the lunar wake is an under-debate process on the lunar plasma sciences (e.g. Futaana et al. 2010; Nishino et al. 2013; Dhanya et al. 2016), and wave excitation in that region has not yet been elucidated. BBNs in the lunar environment were first detected by the Wind spacecraft in the distant wake (Kellogg et al. 1996) and attributed to ion two-stream instability (Ogilvie et al. 1996). However, ESWIs were not found in the waveform data of this distant wake traversal (Kellogg et al. 1996). Electrostatic waves were observed by the ARTEMIS probes during the first lunar wake flybys and attributed to the electron beam mode, but well-defined ESWs were not detected (Tao et al. 2012). More recently, ESWs have been identified by the ARTEMIS probes in a relatively wide area around the Moon including the distant wake, and these ESWs have been explained to be electron holes (Hutchinson and Malaspina 2018; Malaspina and Hutchinson 2019). A recent study by Chu et al. (2021) also showed ESWs during ARTEMIS lunar flybys and attributed them to electron two-stream instability. Most ESWs in the distant wake would be excited in the regions where both ends of the local magnetic field at the spacecraft were connected to the IMF.

Hashimoto et al. (2010) examined the Kaguya LRS/ WFC waveform data obtained at $\sim 120 \mathrm{~km}$ altitude from the lunar surface and found that ESWIs are excited in the lunar wake boundary (which they called Type-A), above magnetic anomalies (Type-B), and over the unmagnetised lunar surface (Type-C). These ESWs in the waveform data appear as BBNs in the electric field spectral data (see Figs. 2, 3, and 4 in Hashimoto et al. 2010). As for the Type-A ESW, Hashimoto et al. (2010) mentioned that the ESWs were excited by the electron two-stream instability between incident solar wind electrons (moving into the wake) and wake electrons accelerated outward by the strong ambipolar electric fields at the wake boundary. They interpreted these ESWs as being excited in the region far from the Moon and propagating to the Kaguya 
orbits. The event occurred when the magnetic field at the spacecraft location was connected to the nightside lunar surface, which reminds us of possible effects of the lunar surface on the electron distributions and the ESW excitation. However, a detailed analysis of electron distribution functions was not performed in their study.

Kaguya observation also revealed that BBNs are generated even in the centre of the deep lunar wake. These BBNs are caused by bi-streaming electron distributions along the magnetic field that result from intrusion of solar wind ions reflected at the lunar surface or crustal magnetic anomalies on the dayside (Type-II entry) (Nishino et al. 2010, 2013). Electron acceleration and BBN generation caused by the Type-II ion entry become evident when the spacecraft location is magnetically detached from the lunar nightside surface (Nishino et al. 2013).

In this paper, we revisit the Hashimoto et al's (2010) Type-A ESW event in the wake boundary on April 2, 2008 , under the condition that the magnetic field at the observed location is connected to the nightside lunar surface. We present electron pitch-angle distributions around the time when the ESW was detected, and discuss interactions between the solar wind strahl electrons and upward-going electrons originating from the nightside lunar surface. A possible role of solar wind ions coming into the wake boundary is also considered.

\section{Instrumentation}

We use plasma, magnetic field and wave data obtained by the Japanese Kaguya (SELENE) spacecraft in orbit around the Moon. Electron and ion data were obtained by PACE (Plasma energy Angle and Composition Experiment) (Saito et al. 2008, 2010). Upward-going electrons were detected by ESA (Electron Spectrum Analyzer)-S1 facing the lunar surface, and downward-going electrons were detected by ESA-S2 looking up space. For ions, we present data from IMA (Ion Mass Analyzer) with a hemispherical field-of-view toward the lunar surface that can detect solar wind ions coming into the wake boundary. The energy ranges used in this study are $24 \mathrm{eV}-9 \mathrm{keV}$ for ESA-S1, $26 \mathrm{eV}-16 \mathrm{keV}$ for ESA-S2, and $18 \mathrm{eV}-28 \mathrm{keV}$ for IMA. The time resolution of electron data from ESA-S1 and S2 used in this study is $16 \mathrm{~s}$, while that of ion data from IMA is $32 \mathrm{~s}$. For the electron measurement, the sensitivity of ESA-S2 was set to be $\sim 4$ times lower than that of ESA-S1 to avoid saturation in the solar wind. However, this situation makes it difficult to compare the downward and upward electron fluxes in the low-density regions such as the lunar wake. Magnetic field data were originally sampled at a $32-\mathrm{Hz}$ resolution by LMAG (Lunar MAGnetometer) (Shimizu et al. 2008; Takahashi et al. 2009; Tsunakawa et al. 2010), and 4-s average data are used in this study. From the PACE and LMAG data, we obtained electron pitch-angle distributions every $16 \mathrm{~s}$ by using time-averaged magnetic field data. Electric field spectrum data were obtained every $8 \mathrm{~s}$ by two antennas named X-channel and Y-channel of Lunar-RaderSounder (LRS)/Wave-Form-Capture (WFC)-H onboard Kaguya (Kasahara et al. 2008; Ono et al. 2010). The two antennas are orthogonal to each other and installed on a plane perpendicular to the nadir direction. The frequency range of WFC-H used in this study is from $1 \mathrm{kHz}$ to $1 \mathrm{MHz}$ which includes the typical BBN frequencies observed by Kaguya in the lunar wake boundary. We exclude the data below $1 \mathrm{kHz}$ because of the highpass filter (HPF) at $1 \mathrm{kHz}$ implemented in the WFC-H receiver, although BBNs might be generated in the lower frequency range in the tenuous environment of the lunar wake. The altitude of Kaguya from the lunar surface was $\sim 110-120 \mathrm{~km}$ for the period of our interest.

We refer to the solar wind data from the Advanced Composition Explorer (ACE) spacecraft (Stone et al. 1998) located far upstream of the Moon. Ion data and 272-eV electron data from Solar Wind Electron, Proton and Alpha Monitor (SWEPAM) (McComas et al. 1998) and magnetic field data from the Magnetic Field Experiment (MAG) (Smith et al. 1998) are used. We also use the solar wind electron and ion data from 3DP (Lin et al. 1995) and magnetic field data from MFI (Lepping et al. 1995) aboard the Wind spacecraft (Acuña et al. 1995).

The Geocentric Solar Ecliptic (GSE) coordinate system, the Selenocentric Solar Ecliptic (SSE) coordinate system, and the ME (Mean Earth/Polar Axis) coordinate system are used.

\section{Observations}

Figure 1 shows an overview of plasma observations by Kaguya from 04:10 to 04:30 UT on April 2, 2008, which include the ESW event at 04:18:33 UT reported by Hashimoto et al. (2010). The Moon's location and Kaguya's orbit for the 20-min period are shown in Fig. 2. For this period, Kaguya travelled northward in the optical shadow from the deep wake $\left(\mathrm{SZA} \sim 157^{\circ}\right.$ at $\left.04: 10 \mathrm{UT}\right)$ to the northern polar region (SZA $\sim 96^{\circ}$ at 04:30 UT). The orbital plane of Kaguya on the day was almost along the noon-midnight meridian plane. The Moon was located at $(36.9,-47.3,-0.6) R_{E}$ in the GSE coordinate system (1 $R_{E}=6378 \mathrm{~km}$, Earth radius), which was far upstream of the Earth's bow shock. Although this location would have been included in the ion foreshock under the typical Parker spiral IMF, a dominant $B_{Z}$ of the IMF prevented the Moon and the spacecraft from being magnetically connected to the bow shock, and indeed no ions reflected at the bow shock were detected at Kaguya's location during the period of our interest. 


\section{Kaguya LRS+LMAG+PACE 2008-04-02 04:10 - 04:30 UT (SSE)}

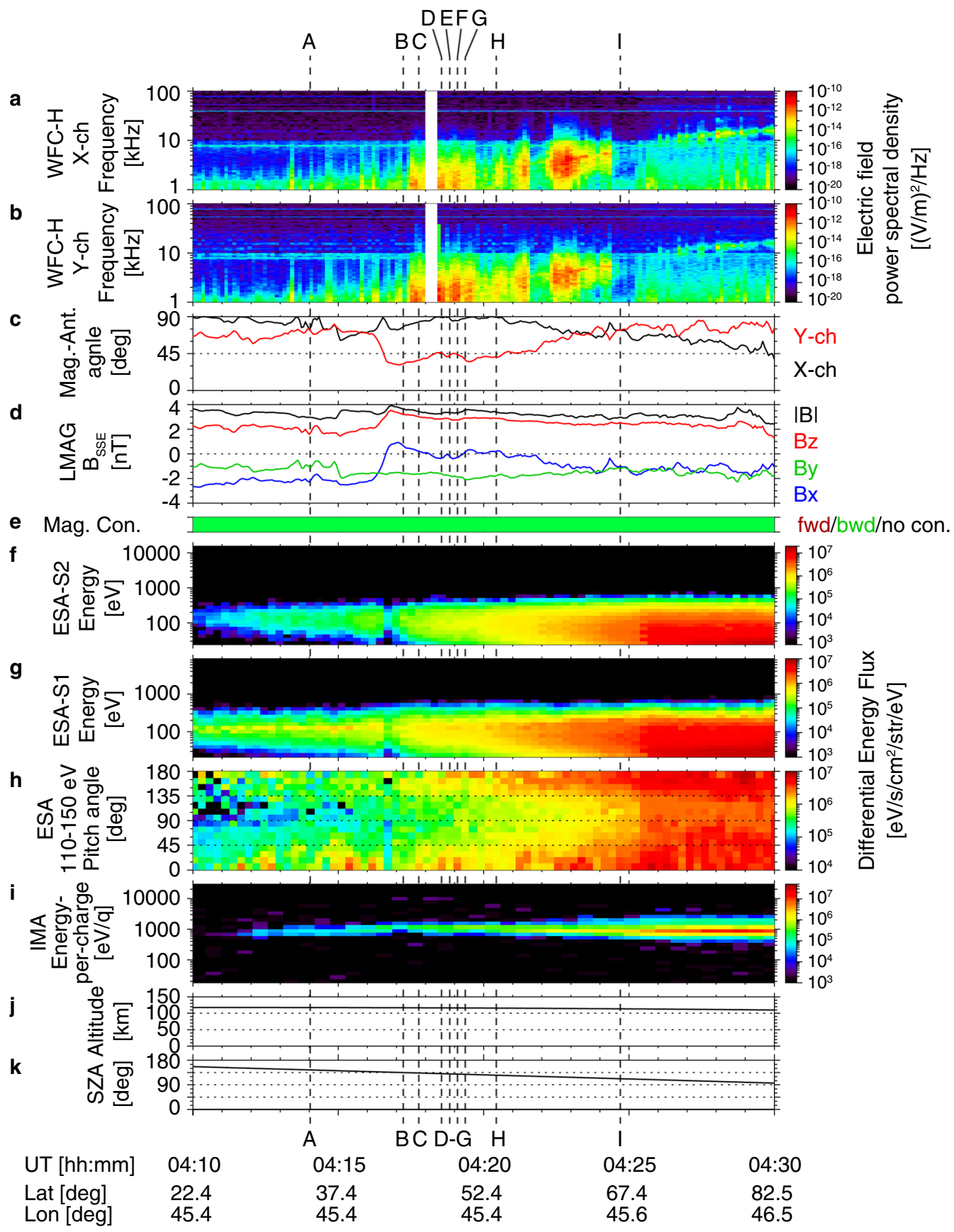

Fig. 1 Kaguya observations from 04:10 to 04:30 UT on April 2, 2008, including an ESW event in the wake boundary studied by Hashimoto et al. (2010). From the top, a frequency-time spectrogram of electric field from X-channel of WFC-H; $\mathbf{b}$ frequency-time spectrogram of electric field from Y-channel; $\mathbf{c}$ angles between the local magnetic field and X-channel and Y-channel antennas; $\mathbf{d}$ magnetic fields in the SSE coordinate system; $\mathbf{e}$ magnetic connection to the lunar surface estimated by a linear extrapolation of the magnetic field at the spacecraft (brown: forward connection (not applicable), green: backward connection, black: magnetically detached (not applicable)); $\mathbf{f}$ electron energy-time spectrogram from ESA-S2; $\mathbf{g}$ electron energy-time spectrogram from ESA-S1; $\mathbf{h}$ pitch-angle time spectrogram from ESA-S1 and ESA-S2; i ion energy-time spectrogram from IMA; $\mathbf{j}$ altitude of Kaguya from the average lunar surface; $\mathbf{k}$ solar zenith angle. Latitude and longitude at the footprint of the spacecraft in the ME coordinate system are presented at the bottom. The vertical dotted lines mark the times when electron pitch-angle distributions in Fig. 1 were taken 


\section{a}
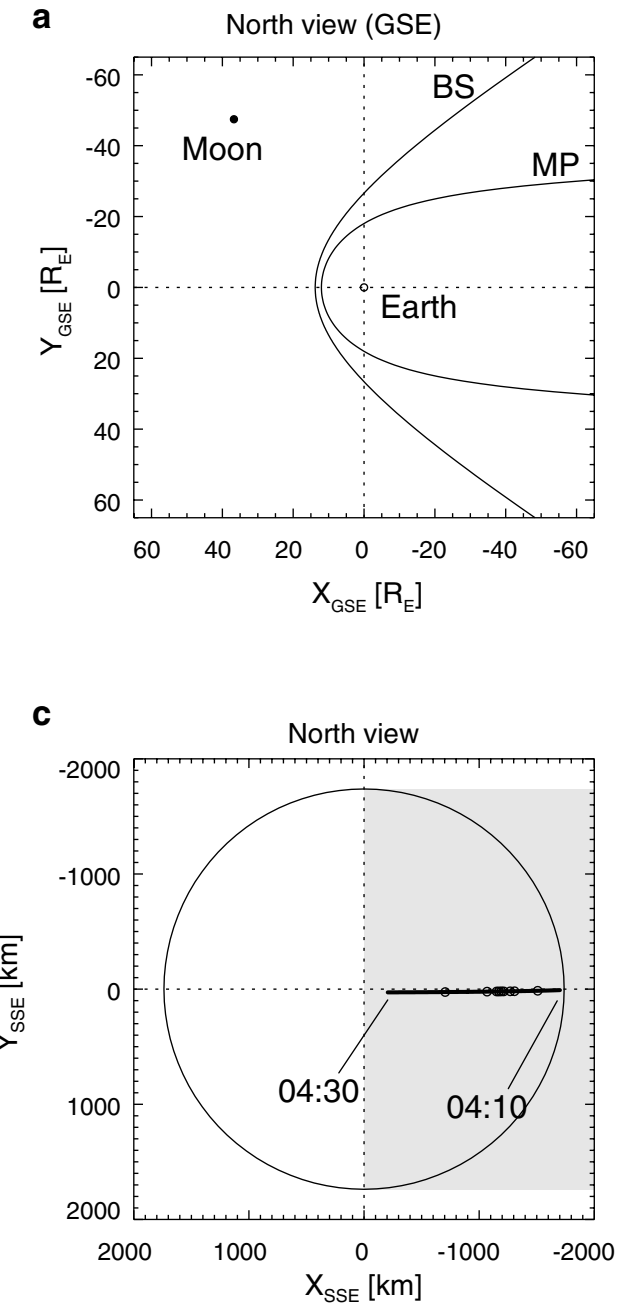

b
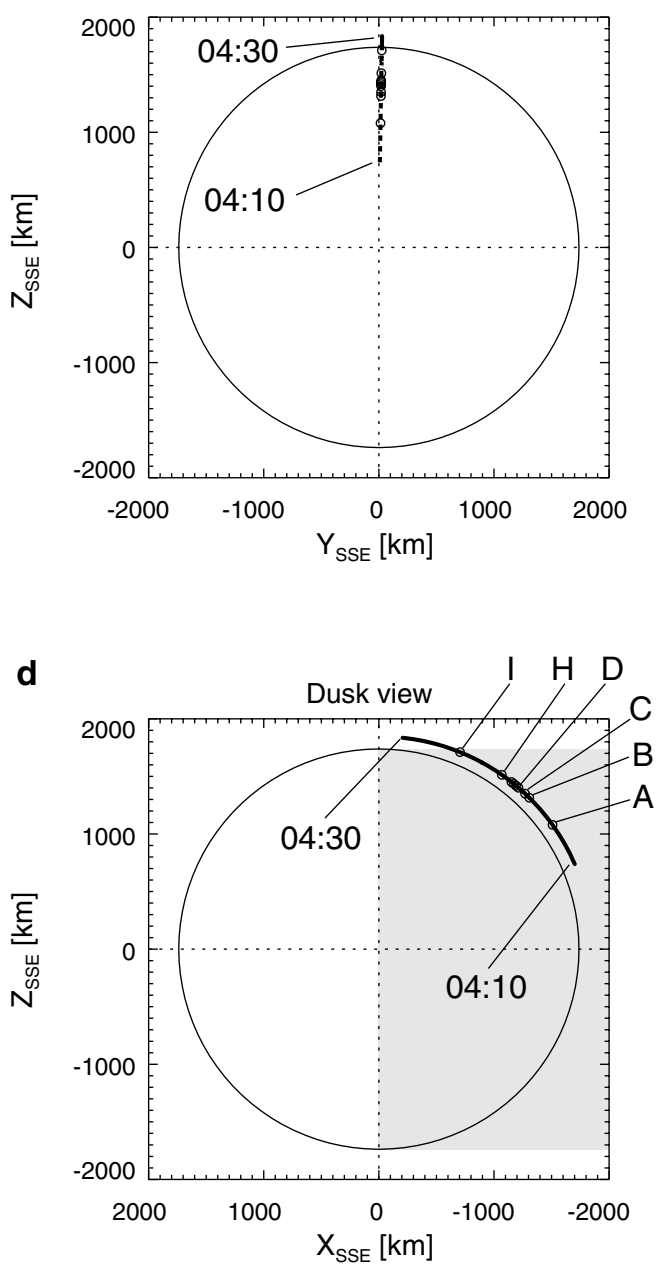

Fig. 2 Kaguya orbit from 04:10 to 04:30 UT on April 2, 2008. a The location of the Moon projected onto the XY plane in the GSE coordinate system, with typical locations of the Earth's magnetopause (MP) for the dynamic pressure of $0.7 \mathrm{nPa}$ (Shue et al. 1998) and the bow shock (BS) (Slavin and Holzer 1981). b-d Kaguya orbit in the SSE coordinate system. The spacecraft locations at the times when the electron pitch-angle distributions in Fig. 3 were taken are also indicated by black points $(\mathbf{a}-\mathbf{i})$

As the spacecraft approached the polar region, electron and ion fluxes gradually increased (Fig. 1f-i), while the BBNs were intermittently detected (Fig. 1a and b) as mentioned later. The IMF had a strength of $\sim 3 \mathrm{nT}$ with a non-negligible northward component throughout this period, while the $B_{X}$ component changes from negative to almost zero around 04:16-04:17 UT with an increase of $B_{Z}$ (Fig. 1d). The electron flux temporarily decreased at the same time as the IMF direction changed, while after 04:18 UT it was larger than before as the spacecraft approached the solar wind. Strong BBNs in the lower frequency range began to be observed at 04:17:28 UT when the spacecraft was located at SZA $\sim 134^{\circ}$. The $32-\mathrm{Hz}$ magnetic field data (not shown) did not present any significant fluctuation during the $\mathrm{BBN}$ events, although the sampling frequency is not high enough to compare them with electric field fluctuations of 1 to a few $\mathrm{kHz}$. In this study, the criterion for a strong $\mathrm{BBN}$ is defined to be $1 \times 10^{-12}(\mathrm{~V} / \mathrm{m})^{2} / \mathrm{Hz}$ in electric field power spectral density in the frequency range between $1 \mathrm{kHz}$ and a few $\mathrm{kHz}$. After a data gap between 04:18:00 and 04:18:24 UT, strong BBNs were detected until 04:19:44 UT with varying intensity. The ESW event at 04:18:33 UT reported by Hashimoto et al. (2010) is included in this period (at the time marked as D in Fig. 1). From 04:17 UT to 04:22 UT the Y-channel antenna was closer to the magnetic field 
direction (hereafter referred to as the parallel direction) than the X-channel antenna (Fig. 1c), and the BBN signals detected by the Y-channel antenna were stronger than those by the X-channel antenna before 04:21 UT. This fact means that the direction of the electric field fluctuations observed before 04:21 UT was closer to the Y-channel antenna than the X-channel antenna. As can be recognised in Fig. 5a of Hashimoto et al. (2010), the electric field fluctuations of the ESW in the perpendicular direction were small but not negligible compared to those in the parallel direction, which might be due to the presence of both the parallel and perpendicular components of the solitary structures as seen in the Earth's polar regions (Franz et al. 2000). Additionally, we note that the BBN signals were safely detected even when the angle between the magnetic field and the antenna was very large $\left(>85^{\circ}\right)$. This is because the power spectral density of BBN is stronger than the background level by several orders of magnitude while amplitudes detected by the antennas depend on the cosine of the angle between the magnetic field and the antenna. After 04:21 UT, the BBNs detected by the $\mathrm{X}$-channel antenna were larger than those by the Y-channel antenna, which means that the perpendicular fluctuations became dominant over the parallel ones. This trend was also observed from 04:22 UT to 04:23 UT when the angle between each antenna and the local magnetic field became close to each other. This change of the electric field directions suggests that the generation mechanism of the BBNs might have changed at around 04:21 UT, although this paper focuses on the BBN generations between 04:17-04:21 UT when the electric field fluctuations were similar to the one reported by Hashimoto et al. (2010).

Typical electron pitch-angle distributions during the wake boundary crossing are shown in Fig. 3. These data were taken at 9 moments marked by the vertical dotted lines in Fig. 1 and black points in Fig. 2. In these distributions, electrons going upward have the pitch angle of $0^{\circ}$ to $90^{\circ}$, and those going downward have the pitch angle of $90^{\circ}$ to $180^{\circ}$. Before strong broadband noises were detected, the upward-going electrons included a very faint beam at $124 \mathrm{eV}$, and no bi-streaming distribution was seen (Fig. 3a). The 124-eV electron beam shows the potential difference between the spacecraft location and the lunar surface caused by negative charging of the nightside lunar surface. (The observation energy at the ESA-S1's energy step of $124 \mathrm{eV}$ ranges from $110 \mathrm{eV}$ to $144 \mathrm{eV}$. See Table 2 in Saito et al. (2010) for details.) Between 04:17:06-04:17:22 UT, after the change in the IMF direction and just before the strong BBN detection, the upward-going electron beam was still faint (Fig. 3b).

The electron pitch-angle distributions during the strong BBN detection are presented in Fig. $3 c$ and d, the latter of which corresponds to the Hashimoto et al's ESW event at 04:18:33 UT. The pitch-angle distributions of $\mathrm{C}, \mathrm{D}$ and $\mathrm{F}$ show a large flux of downward solar wind electrons and an upward electron beam collimated at $124 \mathrm{eV}$. Such strong BBNs were repeatedly observed until 04:19:44 UT, and their intensity appeared to have a period of $\sim 30 \mathrm{~s}$ (judged from Fig. 1a, b). The electron distributions for the period of $\mathrm{E}$ and $\mathrm{G}$ corresponding to weak BBNs show smaller fluxes of upward electron beam at $124 \mathrm{eV}$. The specific values of the differential energy flux of the upward electron beam corresponding to the strong BBNs of C, D and F were $1.6 \times 10^{6}, 2.4 \times 10^{6}$ and $2.9 \times$ $10^{6} \mathrm{eV} / \mathrm{s} / \mathrm{cm}^{2} / \mathrm{str} / \mathrm{eV}$, respectively, while those at $\mathrm{E}$ and G with the weak BBNs were $1.4 \times 10^{6}$ and $1.5 \times 10^{6} \mathrm{eV} / \mathrm{s} / \mathrm{cm}^{2} /$ str/eV, respectively. Since the time resolution of electron observations by ESA-S1 (16 s) is twice as long as that of WFC-H $(8 \mathrm{~s})$, the actual difference in the upward electron beams might be more significant than what is seen in the data. After the strong BBNs ceased at around 04:19:44 UT, weak ones continued to be observed until 04:21 UT. The electrons between 04:20:18-04:20:34 UT, during the weak BBN period, displayed a bidirectional distribution with an upward-going beam at around $158 \mathrm{eV}$ (Fig. 3h), but the beam had a broader distribution in energy space than before. These facts suggest that the BBN generation depends on the distribution of upward electron beams, especially on the temperature of the beam component.

Finally, we present electron pitch-angle distribution between 04:24:34-04:24:50 UT (Fig. 3I) when BBNs were not detected at all. Although the angles between the magnetic field and the antennas at this moment were as large as $74^{\circ}$, we can reasonably conclude that there was no electrostatic wave excitation, because amplitudes detected by the antennas are proportional to the product of the original wave intensity in the parallel direction and a factor of $\cos \left(74^{\circ}\right) \sim 0.28$. Since Kaguya was still inside the lunar optical shadow but coming much closer to the solar wind region (see Fig. 2d), both upward and downward-going electrons had higher fluxes than those observed before with the strong BBNs. In particular, the

(See figure on next page.)

Fig. 3 Electron pitch-angle distributions of 16-s resolution. a 04:13:54-14:14:10 UT, no BBNs; b 04:17:06-04:17:22 UT, no BBNs (or very weak BBNs); c 04:17:38-04:17:54 UT, beginning of strong BBNs; d 04:18:26-14:18:42 UT, strong BBNs, the ESW event reported by Hashimoto et al. (2010); e 04:18:42-04:18:58 UT, weak BBNs; f 04:18:58-04:19:14 UT, strong BBNs; g 04:19:14-04:19:30 UT, weak BBNs; h 04:20:18-04:20:34 UT, weak BBNs; i 04:24:34-04:24:50 UT, no BBNs. For all the data shown here, pitch angels of downward-going (i.e. moonward-going) electrons are between $90^{\circ}$ and $180^{\circ}$, while those of upward-going electrons are between $0^{\circ}$ and $90^{\circ}$ 


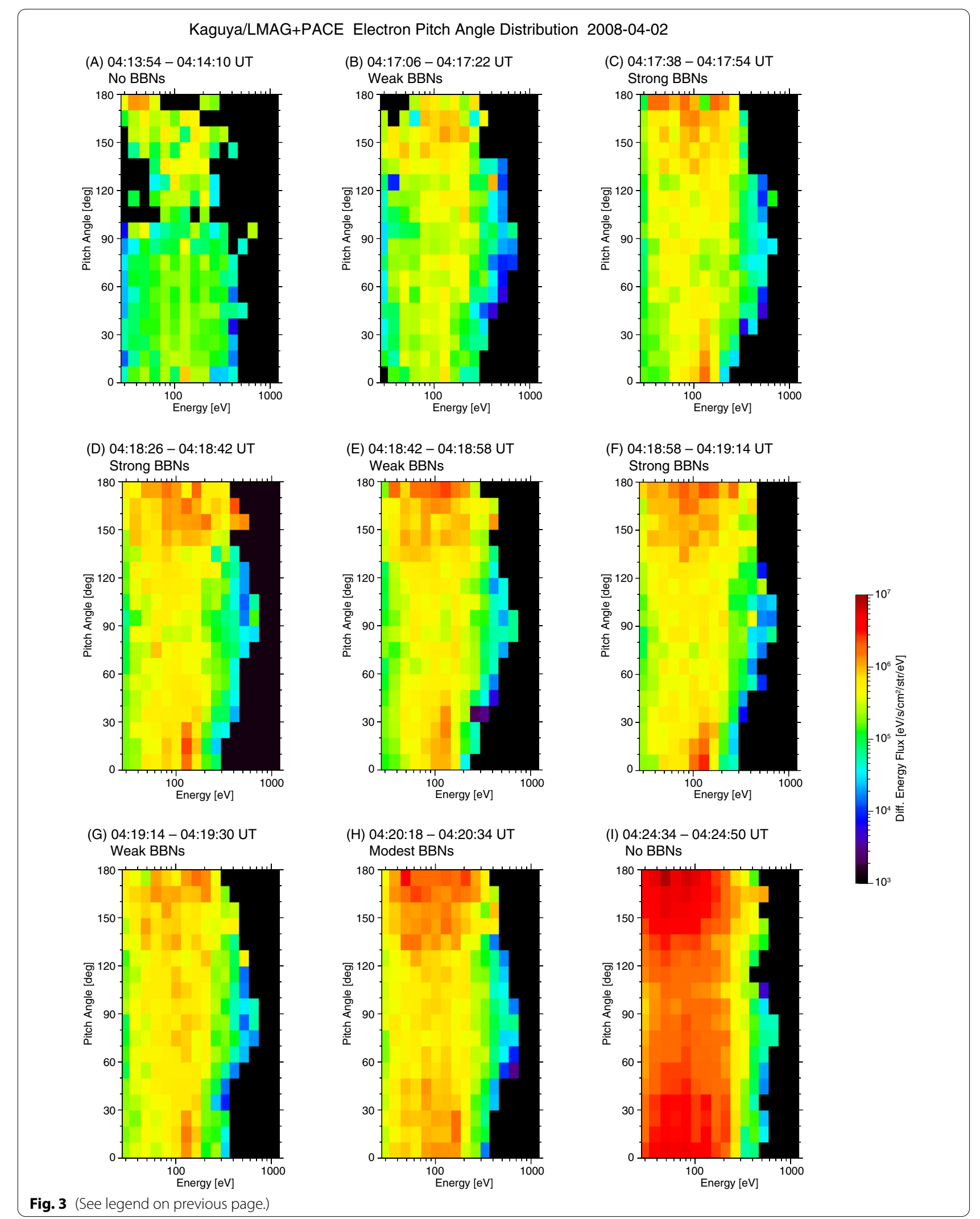


upward-going electrons showed broad distributions in energy space rather than a beam collimated at a narrow energy range. These observations mean that $\mathrm{BBNs}$ are not necessarily excited when the electrons have a bidirectional distribution (Fig. 3f) and imply that the ESW excitation in the wake boundary requires an appropriate amount of incident solar wind electrons and upwardgoing electron beams.

The spacecraft was in the optical shadow of the Moon through the 20-min period, while a non-negligible amount of solar wind ion flux was detected (Fig. 1i). These ions most likely entered the optical shadow region by a combination of their thermal motion itself and Moonward electric fields due to the negative charging of the nightside lunar surface, and possibly partially caused by ambipolar electric fields at the wake boundary. The presence of ions in the wake boundary may facilitate the influx of solar wind electrons and may contribute the generation of BBNs there.

Since the IMF pointed northward through the 20-min period, the location of Kaguya spacecraft travelling in the northern hemisphere was always magnetically connected to the lunar surface (Fig. 1e). This configuration enabled solar wind strahl electrons to precipitate directly onto the nightside lunar surface along the IMF, if they were travelling in the direction antiparallel to the IMF. Figure 4 shows solar wind observations by ACE at $(246,3,-25) R_{E}$ and Wind at $(202,59,-14) R_{E}$ in the GSE coordinate system between 23:00 UT on April 1, 2008 and 05:00 UT on April 2, 2008. The solar wind convection time from ACE to the Moon's location (i.e. $(36.9,-47.3,-0.6) R_{E}$ in GSE) is roughly estimated to be $\sim 55 \mathrm{~min}$, if we consider only the distance in the $X_{G S E}$ direction (209 $R_{E}$ ) and adopt the solar wind flow speed of $\sim 410 \mathrm{~km} / \mathrm{s}$. However, since ACE and the Moon were not aligned with respect to the solar wind flow, it is not easy to shift the ACE data in time simply and compare them with the Kaguya data. Therefore, we focus on the northward IMF starting at around 03:20 UT at ACE, keeping in mind that the ESW event at Kaguya occurred during the strongly northward IMF. Through this period of the northward IMF, the 272-eV electron pitch-angle distribution representing the strahl component of the solar wind electrons displayed a collimated flux in the antiparallel direction to the IMF (Fig. 4a). The electron pitch-angle distributions at $264 \mathrm{eV}$ (Fig. 4f) and $164 \mathrm{eV}$ (Fig. 4g) from Wind also showed continuous flux enhancements in the antiparallel direction, although simple comparison with the Kaguya data is difficult due to a large separation of the two spacecraft in the $Y_{G S E}$ direction. These facts support the idea that the downward-going electrons in the lunar wake boundary observed by Kaguya were indeed strahl component supplied from the solar wind along the IMF, and suggests that the BBN generation in the wake boundary most likely depends on the directions of the solar wind strahl electrons and on the IMF direction.

\section{Discussion}

In this paper, we studied a broadband electric field noise event in the lunar wake traversal observed by Kaguya, focusing on the relationship between BBN generation and electron pitch-angle distributions. We do not have waveform data for the times when the strong BBNs were observed in the wake boundary crossing other than at 04:18:33 UT, but the observed bidirectional electron distributions with BBNs including the ESW event suggest that the electron two-stream instability is a most plausible candidate for the excitation of the ESW. We also point out that the bidirectional electron distribution is not a sufficient condition for generation of BBNs in the lunar wake boundary, and that the pitch-angle distribution of the electron beams going upward from the nightside lunar surface seems to be an essential factor that may control excitation and strength of the ESWs there. The ESW excitation is evident when the upward-going electron beam has a narrower distribution in velocity space. This signature is roughly consistent with simulation results by Omura et al. (1996); Counter-streaming cold electron beams are essential for the ESW excitation in the Earth's magnetotail. On the other hand, the BBN generation after 04:21 UT might result from some process other than the electron two-stream instability, which remains to be clarified in future studies.

ARTEMIS observed similar broadband electric field noises in the lunar wake (Tao et al. 2012), while highfrequency magnetic field fluctuations were not detected by the search coil magnetometer with the sample rate of $\sim 16 \mathrm{kHz}$, which means that the observed broadband noises are electrostatic. Together with the fact that one of the strong broadband noises in the current study corresponds to the ESW period reported by Hashimoto et al. (2010), we suggest that the BBNs observed by Kaguya between 04:17 and 04:21 UT are also likely to be electrostatic waves, although we cannot finally judge whether these waves are electrostatic or electromagnetic due to the limitation of the magnetic field sample rate $(32 \mathrm{~Hz})$.

Here, we estimate the potential of the ESWs found by Hashimoto et al. (2010) and discuss the detectability of electron acceleration. According to Hashimoto et al. (2010), the ESW detected at 04:18:33 UT had a duration of $1 \mathrm{~ms}$ and an amplitude of $2 \mathrm{mV} / \mathrm{m}(4 \mathrm{mV} / \mathrm{m}$ peak-topeak). Since the speed of $124-\mathrm{eV}$ electrons is $\sim 6600 \mathrm{~km} / \mathrm{s}$, the potential $(\phi)$ corresponding to the electrostatic solitary structure is estimated as follows:

$$
\phi=6600 \mathrm{~km} / \mathrm{s} \times 1 \mathrm{~ms} \times 2 \mathrm{mV} / \mathrm{m} \simeq 13 \mathrm{~V} .
$$

The estimated potential is much smaller than the typical energy of the observed electron beams $(>100 \mathrm{eV})$ 


\section{ACE+Wind 2008-04-01 23:00 - 2008-04-02 05:00 UT (GSE)}

a

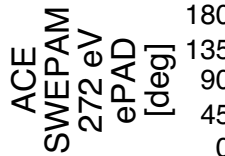

b 岁定出宁

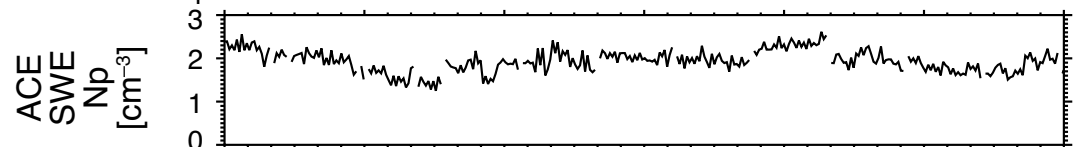

d

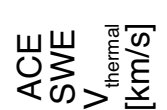

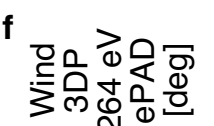
ติ응

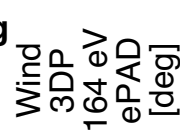

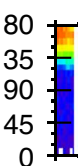
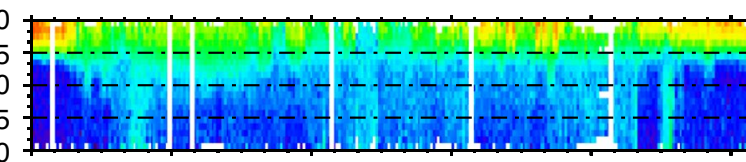

(5)
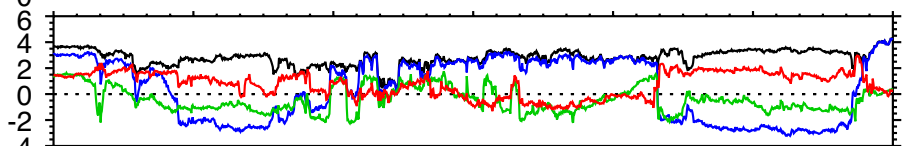

IBI

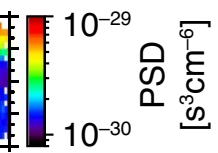

BXGSE

BYGSE

BZGSE

(B)

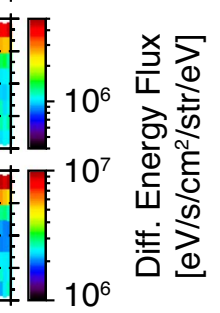

h
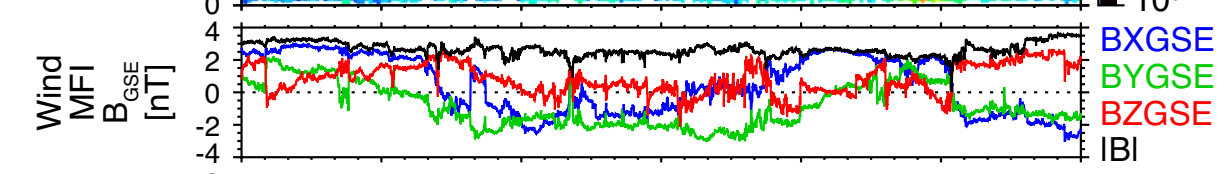

i

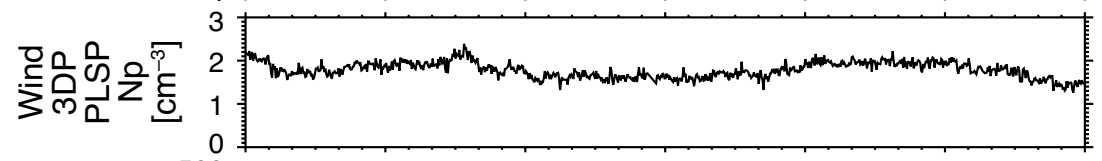

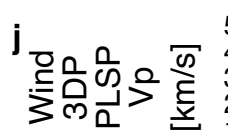

500
400
300
200
100
0

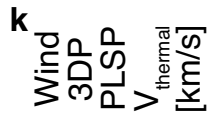

20

UT (hhmm)

$\begin{array}{ll}2300 & 0000 \\ \text { Apr } 01 & \text { Apr } 02\end{array}$

Fig. 4 Solar wind data observed by a-e ACE and f-k Wind spacecraft from 23:00 UT on April 1, 2008 to 05:00 UT on April 2, 2008. From the top, a 272-eV electron pitch-angle distribution in phase-space density; $\mathbf{b}$ magnetic field in GSE; $\mathbf{c}$ ion density; $\mathbf{d}$ ion bulk speed and $\mathbf{e}$ ion thermal speed at ACE, and $\mathbf{f} 264-\mathrm{eV}$ electron pitch-angle distribution; $\mathbf{g}$ 164-eV electron pitch-angle distribution; $\mathbf{h}$ magnetic field in GSE; i proton density; j proton bulk speed and $\mathbf{k}$ proton thermal speed at Wind are plotted. The difference between the GSE and SSE coordinates is negligible in this period

that corresponds to the potential difference between the spacecraft's location and its footprint on the nightside lunar surface. This fact means that small potential structures by ESWs $(\sim 10 \mathrm{~V})$ may be embedded in the large-scale potential structure $(>100 \mathrm{~V})$ predominantly due to negative charging of the nightside lunar surface. 
However, since the estimated potential $\phi$ is smaller than the observation energy range $(110-144 \mathrm{eV})$ at the ESAS1's energy step of $124 \mathrm{eV}$, it is difficult to see an acceleration of electron beams by the electrostatic solitary structures.

We discuss the effects of incident solar wind electrons on the nightside lunar surface when the strong BBNs occurred. By linearly extrapolating the magnetic field observed by Kaguya/LMAG at 04:18:33 UT, we estimated the magnetic footprint to be at $\left(45^{\circ} \mathrm{N}, 42^{\circ} \mathrm{E}\right)$ in the ME coordinate system. At this location on the lunar surface, the crustal magnetic field strength reproduced by the surface vector mapping (SVM) method (Tsunakawa et al. 2015) is as weak as $0.6 \mathrm{nT}$. Based on the simple sum of the crustal magnetic field of $0.6 \mathrm{nT}$ and external magnetic field of $\sim 3 \mathrm{nT}$ observed at Kaguya's location, the magnetic field strength at the footprint is estimated to be at most $3.6 \mathrm{nT}$. This combination of the magnetic field strengths gives a loss cone of $66^{\circ}$ or larger. Therefore, most of the incident solar wind electrons that overcome the potential difference between the Kaguya's location and the nightside lunar surface $(\sim 124 \mathrm{~V}$ in this case) should reach the nightside lunar surface without being reflected either by the crustal magnetic field or by the downward electric field corresponding to the potential difference. The electrons precipitating into the nightside lunar surface may contribute to the negative charging and secondary electron emission there.

Figure 5 schematically summarises the solar wind electron access to the nightside lunar surface along the IMF, negative charging of the nightside lunar surface, upwardgoing electrons, and generation of BBNs. In particular, when the IMF is dominated by a component perpendicular to the solar wind flow, the solar wind strahl electrons can easily access a wide region of the nightside lunar surface. This process results in the negative charging of the nightside lunar surface, which generates Moonward electric fields that accelerate electrons from the nightside surface to higher altitudes to be observed as field-aligned beams. The incident solar wind electrons and the upward electron beams form the bidirectional distributions to generate the BBNs.

We briefly discuss solar wind ion intrusion into the wake boundary and the deeper wake. The ESW event at 04:18:33 UT was observed at $(-1213,20,1401) \mathrm{km}$ in the SSE coordinate system (Fig. 2). The thermal speed of the solar wind protons in the radial (i.e. anti-sunward) direction at ACE was 30 to $40 \mathrm{~km} / \mathrm{s}$ (Fig. 4e), and proton thermal speed at Wind was 40 to $50 \mathrm{~km} / \mathrm{s}$ (Fig. $4 \mathrm{k}$ ). Based on the observations by ACE and Wind, we can expect that some solar wind ions in the faster part of the thermal distribution may have a southward speed higher than $\sim 100 \mathrm{~km} / \mathrm{s}$. Since the bulk solar wind speed in the

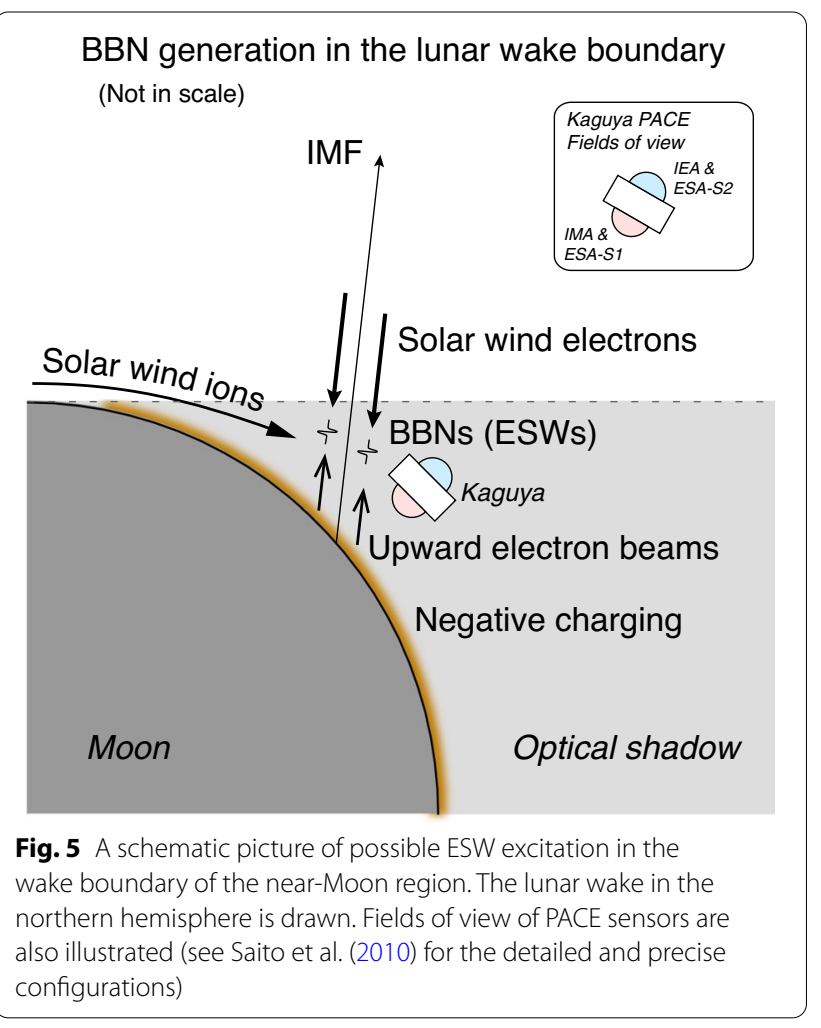

anti-sunward direction was $410 \mathrm{~km} / \mathrm{s}$, it is most likely that the solar wind ion intrusion was mainly caused by the thermal motion. On the other hand, solar wind protons were detected by IMA at around 04:12 UT (SZA $150^{\circ}$ ) in the wake much deeper than the BBN locations. This fact suggests that ambipolar electric fields may also play a role in the solar wind proton intrusion into the deeper wake, as pointed out by Nishino et al. (2009). If the ambipolar electric fields present below the spacecraft altitude, they contribute to upward acceleration of electrons along the magnetic field line. Therefore, upwardgoing electron beams observed at the spacecraft altitude should be generated by combining the Moonward electric field due to the surface charging and the ambipolar electric fields in the wake boundary. The averaged strength of electric fields below the spacecraft calculated from the potential difference $(124 \mathrm{eV})$ and the spacecraft altitude $(\sim 100 \mathrm{~km})$ is in the order of $1 \mathrm{mV} / \mathrm{m}$, which is an-orderof-magnitude stronger than the ambipolar electric fields in the wake estimated in a previous study $[0.3 \mathrm{mV} / \mathrm{m}$ at most (Xu et al. 2019)]. However, the exact location of the ambipolar electric fields and relative strengths of both electric fields remain to be solved.

It is interesting to note that strong BBNs were repeatedly detected with a quasi-period of $\sim 30 \mathrm{~s}$. This quasiperiodic variation of the BBN intensity might correspond to (1) the timescale of negative charging of the nightside 
lunar surface, (2) temporal variation of incident solar wind electron flux or (3) the timescale of heating process of the upward electron beams by wave-particle interactions. However, further studies including analytical treatment and simulation studies of electron two-stream instability are necessary to discuss the possible periodicity of the BBNs in detail.

The generation of the broadband electric field noises reported here may not be limited to the Moon, but is a phenomenon that can generally occur above negatively charged surfaces of heavenly bodies without a thick atmosphere. For example, (1) the planet Mercury whose surface is exposed to high-energy electrons generated in the magnetosphere (Ho et al. 2011, 2012, 2016; Lindsay et al. 2016) and to solar wind energetic electrons (Gershman et al. 2015), (2) Martian moons (Phobos and Deimos), (3) asteroids including Psyche and Didymos, and (4) low-activity comets are candidates for the places of a possible BBN generation by the same mechanism as that in the lunar wake boundary, although different Debye lengths and object sizes may affect the mechanism. Future explorations of the electromagnetic environments of these bodies will give us a clue to a full understanding of the wave-particle interactions on the BBN generations and the possible ESW excitations.

\section{Conclusions}

In this paper, we studied a lunar wake boundary traversal with BBNs in the electric field spectral data observed by Kaguya, focusing on electron pitch-angle distributions around the time of the ESW detection previously reported by Hashimoto et al. (2010). During the wake boundary crossing in the northern hemisphere, solar wind strahl electrons travelling southward came into the wake along the IMF with a strong northward component. The electron pitch-angle distributions showed bi-streaming components of downward-going solar wind strahl electrons and upward-going electron beams originating from the nightside surface. However, the bi-streaming distribution is not a sufficient condition for the strong BBN generation, and the distribution of upward electron beams seems to be correlated with the BBNs. The nightside lunar surface is negatively charged due to the precipitation of solar wind electrons, which generates downward electric fields and accelerate electrons from the nightside surface to higher altitudes along the magnetic field. The solar wind ions most likely come into the wake boundary mainly due to the thermal motions, and the presence of these ions facilitates the influx of solar wind electrons into the region. Ambipolar electric fields in the wake boundary should also contribute to the electron acceleration toward higher altitudes as well as further intrusion of the solar wind ions into the deeper wake. In this way, solar wind ions coming into the wake boundary may play a role in the BBN generation and possible ESW excitation, while further study is necessary to achieve quantitative discussions.

\section{Abbreviations}

ACE: Advanced Composition Explorer; BBN: Broadband noise; CDAWeb: Coordinated Data Analysis Web; ESA: Electron Spectrum Analyser; ESW: Electrostatic solitary wave; GSE: Geocentric solar ecliptic; IMA: Ion Mass Analyser; LRS: Lunar Radar Sounder; IMF: Interplanetary magnetic field; ME: Mean Earth/polar axis; PACE: Plasma energy Angle and Composition Experiment; SSE: Selenocentric solar ecliptic; SVM: Surface vector mapping; SZA: Solar zenith angle; UT: Universal time; WFC: Wave form capture.

\section{Acknowledgements}

The authors are grateful to all members of the SELENE project. The authors gratefully acknowledge the development team of the Space Physics Environment Data Analysis System (SPEDAS) software. The authors also thank the principal investigators of the ACE spacecraft for providing solar wind ion and magnetic field data through CDAWeb at NASA and solar wind strahl electron data through ACE Science Center of California Institute of Technology. The principal investigators of the Wind spacecraft are also acknowledged for providing solar wind plasma and magnetic field data. Hideyuki Usui and Yohei Miyake at Kobe University are acknowledged for providing fruitful discussions. Finally, this Letter is dedicated to the memory of our two colleagues; Prof. Takayuki Ono who passed away in 2013 is acknowledged for his leading the Kaguya/LRS team, and Dr. Yuki Goto who passed away in 2020 is acknowledged for his huge contribution to the development of LRS/WFC-H.

\section{Authors' contributions}

MNN designed this study and prepared the manuscript. YH and YM provided graphical routines. YS and SY contributed to the development, observation, and data processing of PACE. YK, AK, YG and TO contributed to the development, observation, and data processing of LRS/WFC. FT, MM, HShib, HShim and $\mathrm{HT}$ contributed to the development, observation, and data processing of LMAG. All coauthors attended scientific discussion. All authors read and approved the final manuscript.

\section{Funding}

This work was supported by JSPS KAKENHI Grant Numbers 21740359 and 26400477 for MNN.

\section{Availability of data and materials}

Kaguya LRS/WFC, LMAG and PACE data are available from DARTS website at JAXA/ISAS (https://darts.isas.jaxa.jp/). The digital data of the lunar crustal magnetic fields calculated by the SVM method are available from JAXA/JLPEDA website (http://jlpeda.jaxa.jp/globalSVM20150511.zip). Pitch angle distribution of solar wind strahl electrons at $272 \mathrm{eV}$ from ACE/SWEPAM is provided by the ACE Science Center of California Institute of Technology (http://www.srl.calte ch.edu/ACE/ASC/index.html). Solar wind proton data from ACE/SWEPAM, magnetic field data from ACE/MAG, electron and proton data from Wind/3DP and magnetic field data from Wind/MFI are provided by NASA/CDAWeb. The data of the Moon's location were provided by NASA/SSCWeb. Data analysis was performed using the Space Physics Environment Data Analysis System (SPEDAS) V3.1 (see Angelopoulos et al. (2019) in detail).

\section{Declarations}

Ethics approval and consent to participate Not applicable.

\section{Consent for publication}

Not applicable.

\section{Competing interests}

The authors declare that they have no competing interests. 


\begin{abstract}
Author details
${ }^{1}$ Institute of Space and Astronautical Science, Japan Aerospace Exploration Agency, 3-1-1 Yoshinodai, Chuo-ku, Sagamihara, Kanagawa 252-5210, Japan. ${ }^{2}$ Kanazawa University, Kakuma-machi, Kanazawa, Ishikawa 920-1192, Japan. ${ }^{3}$ Kyoto University, Kitashirakawa Oiwake-cho, Sakyo-ku, Kyoto 606-8502, Japan. ${ }^{4}$ Tohoku University, 6-3, Aramaki, Aoba-ku, Sendai, Miyagi 980-8578, Japan. ${ }^{5}$ Osaka University, 1-1 Machikaneyama-cho, Toyonaka, Osaka 560-0043, Japan. ${ }^{6}$ Kyushu University, 744 Moto-oka, Nishi-ku, Fukuoka 819-0395, Japan. ${ }^{7}$ Tokyo Institute of Technology, 2-12-1 Ookayama, Meguro-ku, Tokyo 152-8551, Japan. ${ }^{8}$ Doshisha University, 1-3, Tatara Miyakodani, Kyotanabe, Kyoto 610-0394, Japan. ${ }^{9}$ Earthquake Research Institute, The University of Tokyo, 1-1-1 Yayoi, Bunkyo-ku, Tokyo 113-0032, Japan. ${ }^{10}$ Korea Astronomy and Space Science Institute, 776 Daedeok-daero, Yuseong-gu, Daejeon 34055, South Korea.

${ }^{11}$ Korea University of Science and Technology, 217 Gajeong-ro, Yuseong-gu, Daejeon 34113, South Korea.
\end{abstract}

Received: 30 August 2021 Accepted: 18 December 2021 Published online: 04 January 2022

\section{References}

Acuña MH, Ogilvie KW, Baker DN, Curtis SA, Fairfield DH, Mish WH (1995) The Global Geospace Science Program and its investigations. Space Sci Rev 71:5-21. https://doi.org/10.1007/BF00751323

Angelopoulos V, Cruce P, Drozdov A et al (2019) The Space Physics Environment Data Analysis System (SPEDAS). Space Sci Rev 215:9. https://doi. org/10.1007/s11214-018-0576-4

Chu F, Halekas JS, Cao X, McFadden JP, Bonnell JW, Glassmeier KH (2021) Electrostatic waves and electron heating observed over lunar crustal magnetic anomalies. J Geophys Res 126:e2020JA028880. https://doi.org/ 10.1029/2020JA028880

Dhanya MB, Bhardwaj A, Futaana Y, Barabash S, Alok A, Wieser M, Holmström M, Wurz P (2016) Characteristics of proton velocity distribution functions in the near-lunar wake from Chandrayaan-1/SWIM observations. Icarus 271:120-130. https://doi.org/10.1016/j.icarus.2016.01.032

Franz JR, Kintner PM, Seyler CE, Pickett JS, Scudder JD (2000) On the perpendicular scale of electron phase-space holes. Geophys Res Lett 27:169172. https://doi.org/10.1029/1999GL010733

Futaana Y, Barabash S, Wieser M, Holmström M, Bhardwaj A, Dhanya MB, Sridharan R, Wurz P, Schaufelberger A, Asamura K (2010) Protons in the near-lunar wake observed by the Sub-keV Atom Reflection Analyzer on board Chandrayaan-1. J Geophys Res 115:A10248. https://doi.org/10. 1029/2010JA015264

Gershman DJ, Raines JM, Slavin JA, Zurbuchen TH, Anderson BJ, Korth H, Ho GC, Boardsen SA, Cassidy TA, Walsh BM, Solomon SC (2015) MESSENGER observations of solar energetic electrons within Mercury's magnetosphere. J Geophys Res 120:8559-8571. https://doi.org/10.1002/2015J A021610

Harada Y, Halekas JS (2016) Upstream waves and particles at the Moon. In: Keiling A, Lee DH, Nakariakov $V$ (eds) Low-frequency waves in space plasmas, Geophysical Monograph Series, vol 216. American Geophysical Union, Washington, DC, pp 307-322. https://doi.org/10.1002/9781119055006. ch18

Hashimoto K, Hashitani M, Kasahara Y et al (2010) Electrostatic solitary waves associated with magnetic anomalies and wake boundary of the Moon observed by KAGUYA. Geophys Res Lett 37:L19204. https://doi.org/10. 1029/2010GL044529

Ho GC, Krimigis SM, Gold RE, Baker DN, Slavin JA, Anderson BJ, Korth H, Starr RD, Lawrence DJ, McNutt RL Jr, Solomon SC (2011) MESSENGER Observations of Transient Bursts of Energetic Electrons in Mercury's Magnetosphere. Science 333:1865-1868. https://doi.org/10.1126/science.1211141

Ho GC, Krimigis SM, Gold RE, Baker DN, Anderson BJ, Korth H, Slavin JA, McNutt RL Jr, Winslow RM, Solomon SC (2012) Spatial distribution and spectral characteristics of energetic electrons in Mercury's magnetosphere. J Geophys Res 117:A00M04. https://doi.org/10.1029/2012JA017983

Ho GC, Starr RD, Krimigis SM, Vandegriff JD, Baker DN, Gold RE, Anderson BJ, Korth H, Schriver D, McNutt RL Jr, Solomon SC (2016) MESSENGER observations of suprathermal electrons in Mercury's magnetosphere. Geophys Res Lett 43:550-555. https://doi.org/10.1002/2015GL066850
Holmes JC, Ergun RE, Newman DL, Wilder FD, Sturner AP, Goodrich KA, Torbert RB, Giles BL, Strangeway RJ, Burch JL (2017) Negative Potential Solitary Structures in the Magnetosheath With Large Parallel Width. J Geophys Res 123:132-145. https://doi.org/10.1002/2017JA024890

Hutchinson IH, Malaspina DM (2018) Prediction and observation of electron instabilities and phase space holes concentrated in the lunar plasma wake. Geophys Res Lett 45:3838-3845. https://doi.org/10.1029/2017G L076880

Kasahara Y, Goto Y, Hashimoto K, Imachi T, Kumamoto A, Ono T, Matsumoto $H$ (2008) Plasma wave observation using waveform capture in the Lunar Radar Sounder on board the SELENE spacecraft. Earth Planets Space 60:341-351. https://doi.org/10.1186/BF03352799

Kellogg PJ, Goetz K, Monson SJ, Bougeret JL, Manning R, Kaiser ML (1996) Observation of plasma waves during a traversal of the Moon's wake. Geophys Res Lett 23:1267-1270. https://doi.org/10.1029/96GL00376

Lepping RP, Acũna MH, Burlaga LF et al (1995) The WIND magnetic field investigation. Space Sci Rev 71:207-229. https://doi.org/10.1007/BF00751330

Lin RP, Anderson KA, Ashford S, Carlson C, Curtis D, Ergun R et al (1995) A three-dimensional plasma and energetic particle investigation for the wind spacecraft. Space Sci Rev 71:125-153. https://doi.org/10.1007/ BF00751328

Lindsay ST, James MK, Bunce EJ, Imber SM, Korth H, Martindale A, Yeoman TK (2016) MESSENGER X-ray observations of magnetosphere-surface interaction on the nightside of Mercury. Planetary Space Sci 125:72-79. https://doi.org/10.1016/j.pss.2016.03.005

Malaspina DM, Hutchinson IH (2019) Properties of Electron Phase Space Holes in the Lunar Plasma Environment. J Geophys Res 124:4994-5008. https:// doi.org/10.1029/2019JA026857

Matsumoto H, Kojima H, Miyatake T, Omura Y, Okada M, Nagano I, Tsutsui M (1994) Electrostatic solitary waves (ESW) in the magnetotail: BEN wave forms observed by GEOTAIL. Geophys Res Lett 21:2915-2918. https://doi. org/10.1029/94GL01284

McComas DJ, Bame SJ, Barker P, Feldman WC, Phillips JL, Riley P, Griffee JW (1998) Solar Wind Electron Proton Alpha Monitor (SWEPAM) for the advanced composition explorer. Space Sci Rev 86:563-612. https://doi. org/10.1023/A:1005040232597

Nakagawa T (2016) ULF/ELF waves in near-Moon space. In: Keiling A, Lee DH, Nakariakov V (eds) Low-frequency waves in space plasmas, Geophysical Monograph Series, vol 216. American Geophysical Union, Washington, DC, pp 295-306. https://doi.org/10.1002/9781119055006.ch17

Nishino MN, Maezawa K, Fujimoto M, Saito Y, Yokota S, Asamura K, Tanaka T, Tsunakawa H, Matsushima M, Takahashi F, Terasawa T, Shibuya H, Shimizu $H$ (2009) Pairwise energy gain-loss feature of solar wind protons in the near-Moon wake. Geophys Res Lett 36:L12108. https://doi.org/10.1029/ 2009GL039049

Nishino MN, Fujimoto M, Saito Y, Yokota S, Kasahara Y, Omura Y, Goto Y, Hashimoto K, Kumamoto A, Ono T, Tsunakawa H, Matsushima M, Takahashi F, Shibuya H, Shimizu H, Terasawa T (2010) Effect of the solar wind proton entry into the deepest lunar wake. Geophys Res Lett 37:L12106. https:// doi.org/10.1029/2010GL043948

Nishino MN, Fujimoto M, Saito Y, Tsunakawa H, Kasahara Y, Kawamura M, Matsushima M, Takahashi F, Shibuya H, Shimizu H, Goto Y, Hashimoto K, Omura Y, Kumamoto A, Ono T, Yokota S (2013) Type-II entry of solar wind protons into the lunar wake: Effects of magnetic connection to the nightside surface. Planetary Space Sci 87:106-114. https://doi.org/10.1016/j. pss.2013.08.017

Ogilvie KW, Steinberg JT, Fitzenreiter RJ, Owen CJ, Lazarus AJ, Farrell WM, Torbert RB (1996) Observations of the lunar plasma wake from the WIND spacecraft on December 27, 1994. Geophys Res Lett 23:1255-1258. https://doi.org/10.1029/96GL01069

Omura Y, Kojima H, Matsumoto H (1994) Computer simulation of electrostatic solitary waves: a nonlinear model of broadband electrostatic noise. Geophys Res Lett 21:2923-2926. https://doi.org/10.1029/94GL01605

Omura Y, Matsumoto H, Miyake T, Kojima H (1996) Electron beam instabilities as generation mechanism of electrostatic solitary waves in the magnetotail. J Geophys Res 101:2685-2697. https://doi.org/10.1029/95JA03145

Ono T, Kumamoto Y, Kasahara Y et al (2010) The Lunar Radar Sounder (LRS) Onboard the KAGUYA (SELENE) Spacecraft. Space Sci Rev 154:145-192. https://doi.org/10.1007/s11214-010-9673-8 
Pickett JS (2021) A review of electrostatic solitary wave research from the Cluster mission. J Geophys Res 126:e2021 JA029548. https://doi.org/10. 1029/2021JA029548

Saito Y, Yokota S, Asamura K, Tanaka T, Akiba R, Fujimoto M, Hasegawa H, Hayakawa H, Hirahara M, Hoshino M, Machida S, Mukai T, Nagai T, Nagatsuma T, Nakamura M, Oyama K, Sagawa E, Sasaki S, Seki K, Terasawa T (2008) Low energy charged particle measurement by MAP-PACE onboard SELENE. Earth Planets Space 60:375-386. https://doi.org/10.1186/BF033 52802

Saito Y, Yokota S, Asamura K, Tanaka T, Nishino MN, Yamamoto T, Terakawa Y, Fujimoto M, Hasegawa H, Hayakawa H, Hirahara M, Hoshino M, Machida S, Mukai T, Nagai T, Nagatsuma T, Nakagawa T, Nakamura M, Oyama K, Sagawa E, Sasaki S, Seki K, Shinohara I, Terasawa T, Tsunakawa H, Shibuya H, Matsushima M, Shimizu H, Takahashi F (2010) In-flight performance and initial results of Plasma energy Angle and Composition Experiment (PACE) on SELENE (Kaguya). Space Sci Rev 154:265-303. https://doi.org/ 10.1007/s11214-010-9647-x

Schubert G, Lichtenstein BR (1974) Observations of Moon-plasma interactions by orbital and surface experiments. Rev Geophys 12(4):592-626. https:// doi.org/10.1029/RG012i004p00592

Shimizu H, Takahashi F, Horii N, Matsuoka A, Matsushima M, Shibuya H, Tsunakawa $H$ (2008) Ground calibration of the high-sensitivity SELENE lunar magnetometer LMAG. Earth Planets Space 60:353-363. https://doi.org/ 10.1186/BF03352800

Shue JH, Song P, Russell CT, Steinberg JT, Chao JK, Zastenker G, Vaisberg OL, Kokubun S, Singer HJ, Detman TR, Kawano H (1998) Magnetopause location under extreme solar wind conditions. J Geophys Res 103:1769117700. https://doi.org/10.1029/98JA01103

Slavin JA, Holzer RE (1981) Solar wind flow about the terrestrial planets 1. Modeling bow shock position and shape. J Geophys Res 86:11401-11418. https://doi.org/10.1029/JA086iA13p11401

Smith CW, Acuña MH, Burlaga LF, L'Heureux J, Ness NF, Scheifele J (1998) The ACE Magnetic Field Experiment. Space Sci Rev 86:613-632. https://doi. org/10.1023/A:1005092216668

Stone EC, Frandsen AM, Mewaldt RA, Frandsen AM, Mewaldt RA, Christian ER, Margolies D, Ormes JF, Snow F (1998) The advanced composition explorer. Space Sci Rev 86:1-22. https://doi.org/10.1023/A:1005082526 237

Takahashi F, Shimizu H, Matsushima M, Shibuya H, Matsuoka A, Nakazawa S, lijima Y, Otake H, Tsunakawa H (2009) In-orbit calibration of the lunar magnetometer onboard SELENE (KAGUYA). Earth Planets Space 61:1269-1274. https://doi.org/10.1186/BF03352979

Tao JB, Ergun RE, Newman DL, Halekas JS, Andersson L, Angelopoulos V, Bonnell JW, McFadden JP, Cully CM, Auster HU, Glassmeier KH, Larson DE, Baumjohann W, Goldman MV (2012) Kinetic instabilities in the lunar wake: ARTEMIS observations. J Geophys Res 117:A03106. https://doi.org/ 10.1029/2011JA017364

Tsunakawa H, Shibuya H, Takahashi F, Shimizu H, Matsushima M, Matsuoka A, Nakazawa S, Otake H, lijima Y (2010) Lunar magnetic field observation and initial global mapping of lunar magnetic anomalies by MAP-LMAG onboard SELENE (Kaguya). Space Sci Rev 154:219-251. https://doi.org/10. 1007/s11214-010-9652-0

Tsunakawa H, Takahashi F, Shimizu H, Shibuya H, Matsushima M (2015) Surface vector mapping of magnetic anomalies over the Moon using Kaguya and Lunar Prospector observations. J Geophys Res 120:1160-1185. https:// doi.org/10.1002/2014JE004785

Xu S, Poppe AR, Halekas JS, Mitchell DL, McFadden JP, Harada Y (2019) Mapping the Lunar Wake Potential Structure With ARTEMIS Data. J Geophys Res 124:3360-3377. https://doi.org/10.1029/2019JA026536

\section{Publisher's Note}

Springer Nature remains neutral with regard to jurisdictional claims in published maps and institutional affiliations.

\section{Submit your manuscript to a SpringerOpen ${ }^{\circ}$ journal and benefit from:}

- Convenient online submission

- Rigorous peer review

- Open access: articles freely available online

- High visibility within the field

- Retaining the copyright to your article

Submit your next manuscript at $\boldsymbol{\nabla}$ springeropen.com 